\title{
Effect of ball end milling parameters on surface and subsurface of Inconel-718
}

\author{
Nandkumar N. Bhopale *, Raju S. Pawade \\ Department of Mechanical Engineering, Dr.Babasaheb Ambedkar Technonogical University, Lonere- 402103, MS, India \\ *Corresponding author E-mail: bhopalen@yahoo.com
}

Copyright () 2015 Nandkumar N. Bhopale and Raju S. Pawade. This is an open access article distributed under the Creative Commons Attribution License, which permits unrestricted use, distribution, and reproduction in any medium, provided the original work is properly cited.

\begin{abstract}
Inconel-718 has poor machinability due to this; it is leading to severe low surface integrity. The paper discusses the effect of ball end milling parameters on surface integrity produced during dry and chilled machining environment. In this work, the variable depth of cut concept of Scattergood is used to describe the influence of machining process parameters on the machined surface. Taguchi orthogonal (L8) experiments were designed considering five input parameters. The surface integrity in terms of arithmetic average surface roughness Ra and the microhardness is analysed in this paper. The results show that the dry machining was beneficial for surface integrity.
\end{abstract}

Keywords: Ball End Mill; Environment; HSM, Inconel-718; Microhardness.

\section{Introduction}

Inconel-718 is one of the proven high-performance materials, which can withstand stringent operating conditions in aero-engine, gas turbine and defense components. It finds applications in aero-engine in the manufacture of flexible web parts like rings and blades [1-2]. However, higher specific shear strength and poor thermal conductivity of these alloys causes difficulties during machining. Higher tool wear on account of rapid work hardening during machining promotes larger tool wear and therefore show poor surface integrity of after machining [3-7]. A number of researchers have worked on the experimental as well as mathematical modeling of ball end milling process. There are attempts to identify optimum processing parameters combination that gives the best surface finish. Moreover, most of these studies are related to generation of plane surfaces using radial feed in a ball end milling. Aspinwall et al. [8] found a good surface finish during high speed ball end milling of Inconel-718 plate. Further, the experimental analysis with cutter orientation, and lead/tilt angles during five-axis ball end milling of turbine blade has shown that the best surface finish is obtained during milling in horizontal inward direction with a $15^{\circ}$ tilt angle with respect to vertical axis. Bouzakis et al. [9] have evaluated the undeformed chip geometry and cutting forces using initial part geometry, tool path and tool geometry as input parameters. Further, they have shown that the chip width and thickness of the cutting edge at its successive revolving positions are a function of two flute ball end mill in the case of down milling. Sonawane and Joshi [10] demonstrated the influence of process parameters on surface topography during ball end milling. The machined surfaces show formation of distinct bands along the periphery of cutter edge. They found the influence of chip compression and instantaneous shear angle on surface roughness and microhardness in those bands. Mizugaki et al. [11] have theoretically determined the machined surface profile. They developed mathematical equations to identify the cusp height at any point on the machined surface. Shokrani et al. [12] have shown that the cryogenic cooling produced better machined surface as compared to that of dry machining during ball end milling. Kasim et al. [13] a mathematical model is established to predict the location of pitting on the PVD coated carbide milling cutter. Dosbaeva et al. [14] examined the machined surface cross sections and found the origin of defect formation after machining. The model will be useful to determine the exact reason of nothing and flaking of the milling cutter. Kaya et al. [15] developed ANN based flank wear simulation under changing cutting conditions, using cutting time, and cutting force. Therefore, it appears from the literature review that most of the researchers have determined the effects of various machining conditions on the cutting 
force and surface quality in ball end milling process. They have predicted the tool workpiece interactions, chip geometry, cutting forces and surface topography using analytical as well as experimental models. Further, literature review shows that the mechanism and mechanics of surface generation and resultant surface integrity of ball end milling of Inconel-718 have received limited attention. As mentioned above, the objectives of the work is to study the effect of process parameters on surface quality after high speed ball end milling of Inconel-718, in terms of surface roughness (Ra), surface damage and microhardness of machined surface and subsurface. The number of passes and the machining environment has also been considered in this investigation.

\section{Experimental work}

\subsection{Experimental theme and design}

In line with the objectives of the work, the selected response variables are surface roughness and the subsurface induced microhardness after machining. Further, the surface alterations were qualitatively analysed to understand the surface topography. The range of cutting conditions for this work was based on initial experiments and tool catalogue [16], see Table 1.

Table 1: Control Factors and Their Levels for Experiment

\begin{tabular}{lll}
\hline \multirow{2}{*}{ Control factors } & \multicolumn{2}{c}{ Level } \\
\cline { 2 - 3 } & 1 & 2 \\
\hline Cutting speed (m/min) & 25 & 75 \\
Feed (mm/tooth) & 0.06 & 0.12 \\
Axial depth of cut (mm) & 0.50 & 1.00 \\
Environment & Dry & Chilled air \\
Cutter orientation & Up milling & Down milling \\
Number of pass (Nos.) & 1 & 2 \\
\hline
\end{tabular}

Table 2: Processing Conditions for Experimental Runs

\begin{tabular}{|c|c|c|c|c|c|c|}
\hline \multirow[b]{2}{*}{ Test Run } & \multicolumn{6}{|c|}{ Input Factors } \\
\hline & Environment & $\begin{array}{l}\text { Cutting speed } \\
(\mathrm{m} / \mathrm{min})\end{array}$ & $\begin{array}{l}\text { Feed } \\
\text { (mm/tooth) }\end{array}$ & Axial depth of cut (mm) & Cutter path & Nos. Of pass \\
\hline 1 & Dry & 25 & 0.06 & 0.5 & $\mathrm{Up}$ & 1 \\
\hline 2 & Dry & 25 & 0.06 & 1.0 & Down & 2 \\
\hline 3 & Dry & 75 & 0.12 & 0.5 & Up & 2 \\
\hline 4 & Dry & 75 & 0.12 & 1.0 & Down & 1 \\
\hline 5 & Chilled air & 25 & 0.12 & 0.5 & Down & 1 \\
\hline 6 & Chilled air & 25 & 0.12 & 1.0 & Up & 2 \\
\hline 7 & Chilled air & 75 & 0.06 & 0.5 & Down & 2 \\
\hline 8 & Chilled air & 75 & 0.06 & 1.0 & Up & 1 \\
\hline
\end{tabular}

(a)

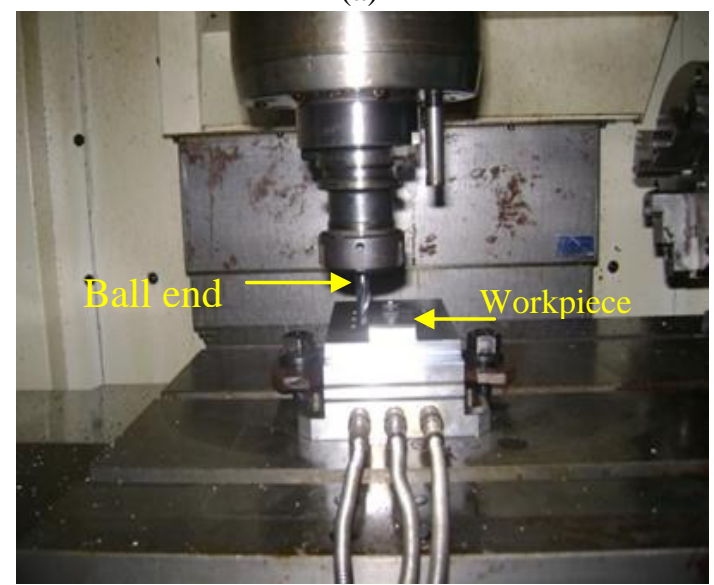

Fig. 1: (a) Close up View of Ball End Milling, and (b) Vortex Tube Refrigerator for Chilled Air Supply (b)

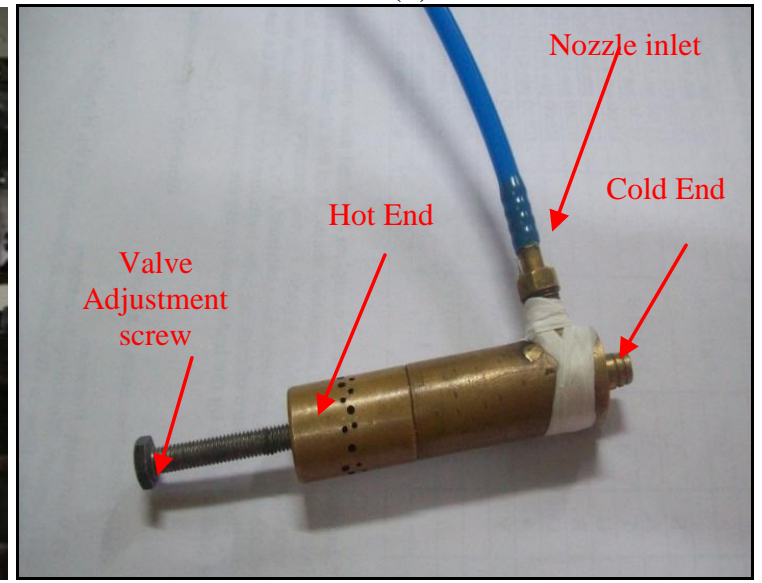




\subsection{Work piece preparation and experimental setup}

The experiments were performed on Inconel-718 specimen of dimensions $160 \mathrm{~mm} \times 70 \mathrm{~mm} \times 8 \mathrm{~mm}$ in length, width and thickness, respectively (Fig.1). The chemical composition of work material is $51.3 \% \mathrm{Ni}, 20.14 \% \mathrm{Fe}, 18.17 \% \mathrm{Cr}$, $4.8 \% \mathrm{Nb}, 3.25 \% \mathrm{Mo}$, and balance C. Solid carbide Ti-Al-N coated ball end mill cutters of 10 mm diameter, 100 rake angle and 300 helix angle with two cutting flutes were used for the experiments. A vertical CNC milling machine (HASS, Model-TM2) was used to carry out the experiments in dry and chilled air environments (Fig.1). The experiments were performed at various machining conditions as per the L8 experimental array [17] as shown in Table 2. Each experiment was replicated once. The machined surface roughness was examined and recorded using Mitutoyo make, Surftest- SJ 301 portable surface roughness tester. The chilled air was supplied to the machining region by using vortex tube refrigerator fabricated in house (see Fig.1b). After machining, each work specimen was cut into three segments across the machined surface of size $15 \mathrm{~mm} \times 15 \mathrm{~mm} \times 10 \mathrm{~mm}$ using a precision saw for measurement of the surface roughness, surface damages and microhardness. The work specimen segments for the measurement of microhardness were hot mounted in bakelite, and polished carefully to obtain a scratch free surface. For this purpose, initially the manual polishing using coarse, intermediate and fine polishing was carried out with water proof SiC polishing papers. Further, the hand polished specimens were cloth polished on a polishing machine using Al2O3 powder and water to obtain fine mirror like surface. Microhardness was measured on the transverse section of the machined surfaces at depths ranging from $25 \mu \mathrm{m}$ to $3000 \mu \mathrm{m}$ from the respective sample edges (see Fig.4). The microhardness beneath the machined surface was examined by Vickers indenter at a load of 100 gf.-f for 15 seconds, using a square-base pyramid, whose opposite sides meet at the apex at an angle of 1360 on Vickers hardness tester (Make Shimadzu, Model-MV2). A bakelite fixture was used to fix the mount on table to ensure accurate measurement. Surface microhardness measurements were taken at different points at same level. An average of three measurements was taken as final surface microhardness. During the measurement, an adequate spacing between the indentations was provided to avoid overlap.

\section{Results and discussion}

Experimental results were analysed in terms of understanding of the surface damage, surface roughness and microhardness profile beneath the machined surface.

\subsection{Analysis of surface topography}

SEM microscopy of the machined surface was also performed to analyse the surface topography, which clearly reveals the surface irregularities caused due to machining. Even though the surface appears smooth, there may be many surface irregularities which can be noticed in high magnification microscopy. A typical topography shows the presence of different surface bands as the depth of cut/chip cross-section area varies, see Fig.2 (a-b). An arrow in the figures indicates the width of the bands identified on the machined surfaces (Fig.2. a-b). The first band is just under the tool tip and is rough it is followed by a smooth and again by a rough band. This indicates that there is a significant effect of depth of cut on machined surface topography which is more irregular due to vibration effect. A narrow rubbing zone just under the tool tip is considered as a part of band \#1 (Fig.2. a). All the four band regions on the machined surface are magnified so that the surface topography can be observed in detail in Fig.2 (a-b). The surface scallops with higher surface irregularities are clearly observed in band \#1. The minimum variation is shown in the band \#2 and band \#3. SEM photographs of machined surfaces during dry machining and chilled air machining are shown in Fig.2 (b). In general, more surface damage is observed in band \#1. It is noticed that the width of bands also changes with a change in machining conditions. Machining with a dry environment is found to be better as it introduces less alteration on the machined surfaces.

It is now clear that the ball end milling cutter geometry generates a complex surface topography consisting of bands on the machined surfaces. The magnitude of width of band changes with a change in feed, depth of cut and tool geometry parameters [10]. 


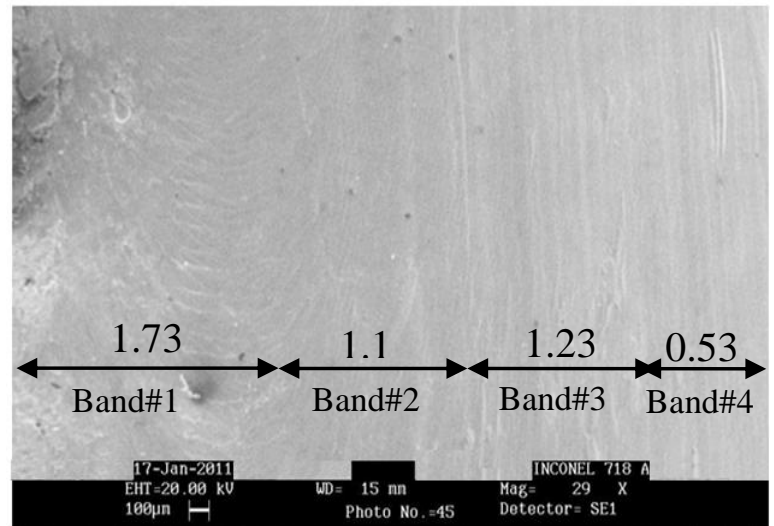

$V c=25 \mathrm{~m} / \mathrm{min}, f=0.06 \mathrm{~mm} /$ tooth, $a p=0.5 \mathrm{~mm}, a_{p}=1 \mathrm{~mm}$, Up milling, Environment $=$ dry, Number of pass $=1$

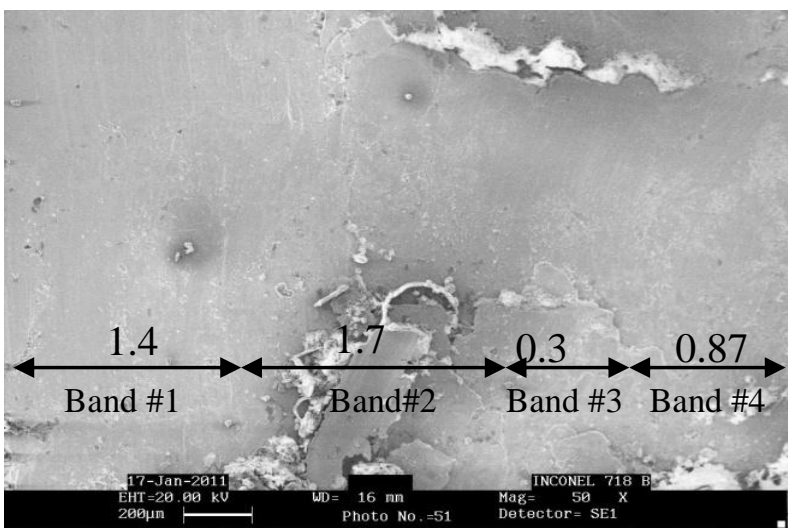

$V c=75 \mathrm{~m} / \mathrm{min}, \mathrm{f}=0.06 \mathrm{~mm} /$ tooth $, a p=0.5 \mathrm{~mm}, a_{p}=1 \mathrm{~mm}$, Up milling, Environment $=$ chilled, Number of pass $=1$

Fig. 2: (a-b) SEM Micrographs of Machined Surface with Different Bands at Various Machining Conditions

\subsection{Quantitative analysis of surface roughness}

In addition, the examination of surface topography was carried out in terms of arithmetic average and is discussed below surface roughness $(\mathrm{Ra})$.

Surface Roughness assessment on different bands, it is clearly understood from Fig.3 that the surface generated in band \#1 in all the experimental conditions show higher Ra values as compared to that with other bands \#2 to \#4. The initial input of the cutter leads to vibrations and chatter that might produce rough surface in this band. In the case of surface roughness almost all the chosen machining parameters influence on the surface roughness in the case of band \#1 only. The surface roughness in band \#1 region is more influential as compared to other bands. This might be due to the dominant effect of tool geometry on the machined surface generation near the tool tip area as compared to the regions away from the tool tip during machining. It is examined that the value of surface roughness (Ra) was low when the milling was carried out in dry machining environment. A change in machining environment from dry to chilled air due to that a considerable surface roughness ( $\mathrm{Ra}$ ) value increases. The thermal stress experienced by the rapid change in environment from dry to chilled causes significant chipping of the machined surfaces. It is noticed that the small amount of increment in surface roughness with an increase in the depth of cut and due to this the chip thickness increases, which interrupts the process leading to intensive material deformation of the machined surface and thus shows higher surface roughness (Ra) at higher feed and the chip is not removed completely for the machined surface near the tool tip region leaving behind a surface irregularity and due to this surface roughness is higher.

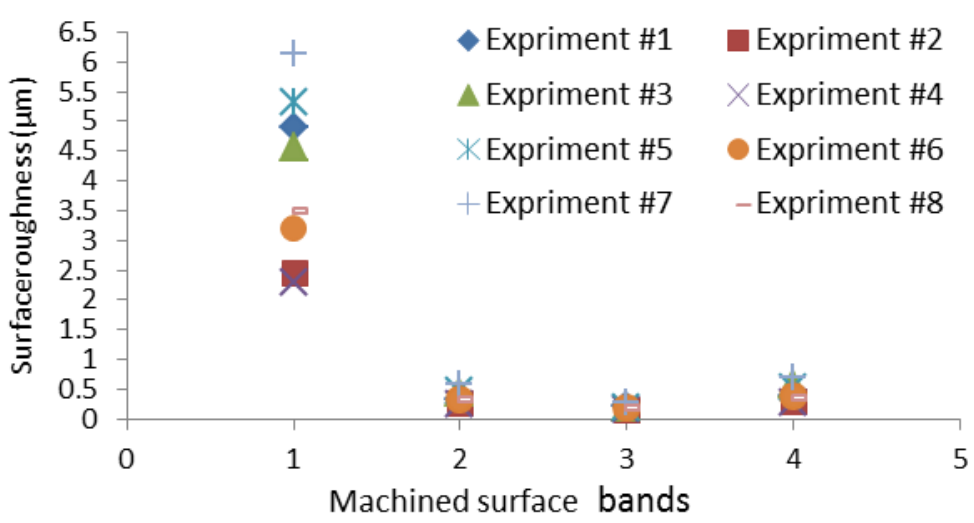

Fig. 3: Comparison of Surface Roughness at Different Bands at Various Machining Conditions

In case of cutting speed, it is observed that the surface roughness decreases with an increase in the cutting speed 25 $\mathrm{m} / \mathrm{min}$ to $75 \mathrm{~m} / \mathrm{min}$. The contact duration with the cutter and workpiece is less at a higher cutting speed, which prevents the chip interruption with the cutter and hence the surface roughness is low at higher cutting speed. However, the surface roughness increases by a small amount during up milling process. During down milling operation, smooth machining was observed due to absence of vibration. However, vibration is more likely to occur during up milling in which the thrust force acts on the $\mathrm{z}$ axis, which resulting into greater tendency for chatter as the majority of the thrust force pushes cutter away from the surface. 


\subsection{Analysis of subsurface deformation}

Ball end milling operation produces significant amount of thermal as well as mechanical properties changes at beneath the machined surface due to generation of high temperature and stresses during machining of Inconel-718 [18]. The subsurface and near surface layer beneath the machined surface undergo deformation and hence show changes in the mechanical properties such as alteration in the hardness values along the cross section beneath machined surface [19] , (see Fig.4). Shows the scheme of microhardness measurement whereas, the Fig.4 shows typical measurement of microhadness that are taken along the cross section of machined surface. A variation of subsurface microhardness beneath the machined surface for few specimens is shown in Fig.5. The corresponding results are discussed in this section. It is found that the maximum microhardness occurs at the region near the first and, as we move from region of band \#1 to band \#4, the microhardness decreases. This can be attributed to the fact that the rubbing forces at band \#1 due to the minimum cutting speed in the tool tip area (band \#1) causes more mechanical loads in the band \#1 region [20]. The microhardness also shows different patterns under various cutting parameters. It is observed that various machining parameters such as higher cutting speed, chilled environment, two passes and up milling, the workpiece surface shows more microhardness values as than that of with the other machining conditions (Fig. 5).

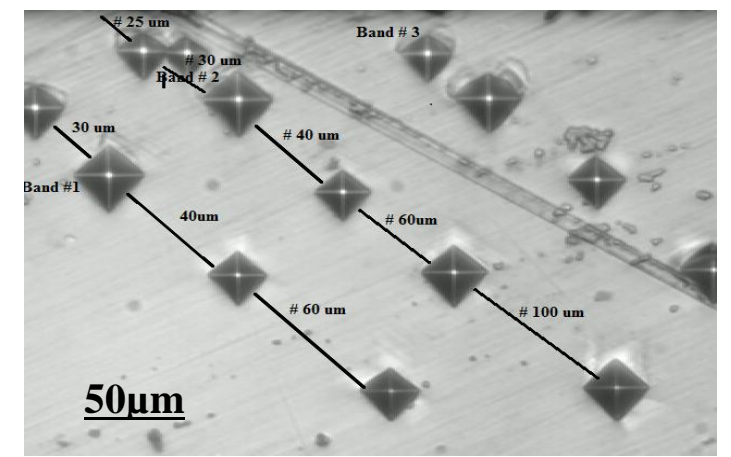

Fig. 4: Actual Photograph of Microhardness Indentation beneath the Surface

However, at lower cutting speed, with the dry environment, the workpiece shows lower hardness values (Fig.5). The microhardness is higher on the layers very close to the machined surface (at \#30 $\mu \mathrm{m}$ depth) in all the bands. It is decreases with the depth of machined surface, due to a decrease in the work hardening of the material beneath the surface layer. It is again observed in all microhardness profiles (Fig.5) that after certain depth beneath the surface layer, the bulk hardness (400-450 Hv) is achieved. Therefore, the machining affected zone (MAZ) is defined as the depth below the subsurface, where the microhardness reaches to the bulk hardness values. In all the surface bands, the microhardness in band \#1 appears to be the maximum followed by in the band \#2 and so on in all the experiments. The maximum microhardness value, i.e. $958 \mathrm{Hv}$ is observed at band \#2 in experiment \#5 (Fig.5), which was performed at higher cutting speed, the chilled environment, two passes with up milling and at lower feed as well as depth of cut values. On the other hand, the lowest microhardness value, i.e. $490 \mathrm{Hv}$ is observed at band \#1 in experiment \#4 (Fig.5), which was performed at higher cutting speed, feed and depth of cut in dry environment, down cutter path position with a single pass. As the band \#2 and band \#3 have relatively better surface finish, various microhardness values in this region at different machining conditions are compared and shown in (Fig.5). It is observed that the machining parameter shows significant effect on the microhardness in all bands. Among the selected control factors the machining environment has shown dominant effect on the microhardness at beneath the machined surface. It is noted that the microhardness value is low when the milling was carried out in dry machining environment beneath $25 \mu \mathrm{m}$. A change in machining condition from dry to chilled air causes an increment in the microhardness value. This steep increase in the microhardness value for band \#1, \#2 and \#3 is due to the higher mechanical load due to prevailing quenching effect in the presence of chilled environment that leads to workhardning of the machined surface. Among all these bands, the effect of the increase in workhardening is more predominant in the case of band \#1 region. The temperature in the machining zone increases with an increase in the cutting speed that could lead to thermal softening of the machined surface and subsurface layer. This phenomenon is observed in the regions of band \#1, \#3 and \#4. However, the effect is opposite in the band \#2 region in that case the machined surface shows an increased microhardness value. In the region near tool tip i.e. band \#1, the cutting tool contacts over a smaller surface area due to which more amount of frictional heat gets concentrated in this region of band \#1 and hence the effect of thermal load is more as compared to mechanical loading. However, in the case of region of band \#2, the frictional heat gets distributed over a large surface area beneath the cutter edge and thus the effect of thermal load becomes less prominent and therefore mechanical work hardening showing higher values of microhardness. The effect of number of passes on the machined surface microhardness, it is noticed that there is a significant increase in microhardness during two-pass machining as compared to single-pass machining. The machined surface becomes work hardened after the first pass due to rapid work hardening tendency of Inconel-718. Therefore, the two-pass machining always shows increased value of microhardness. 

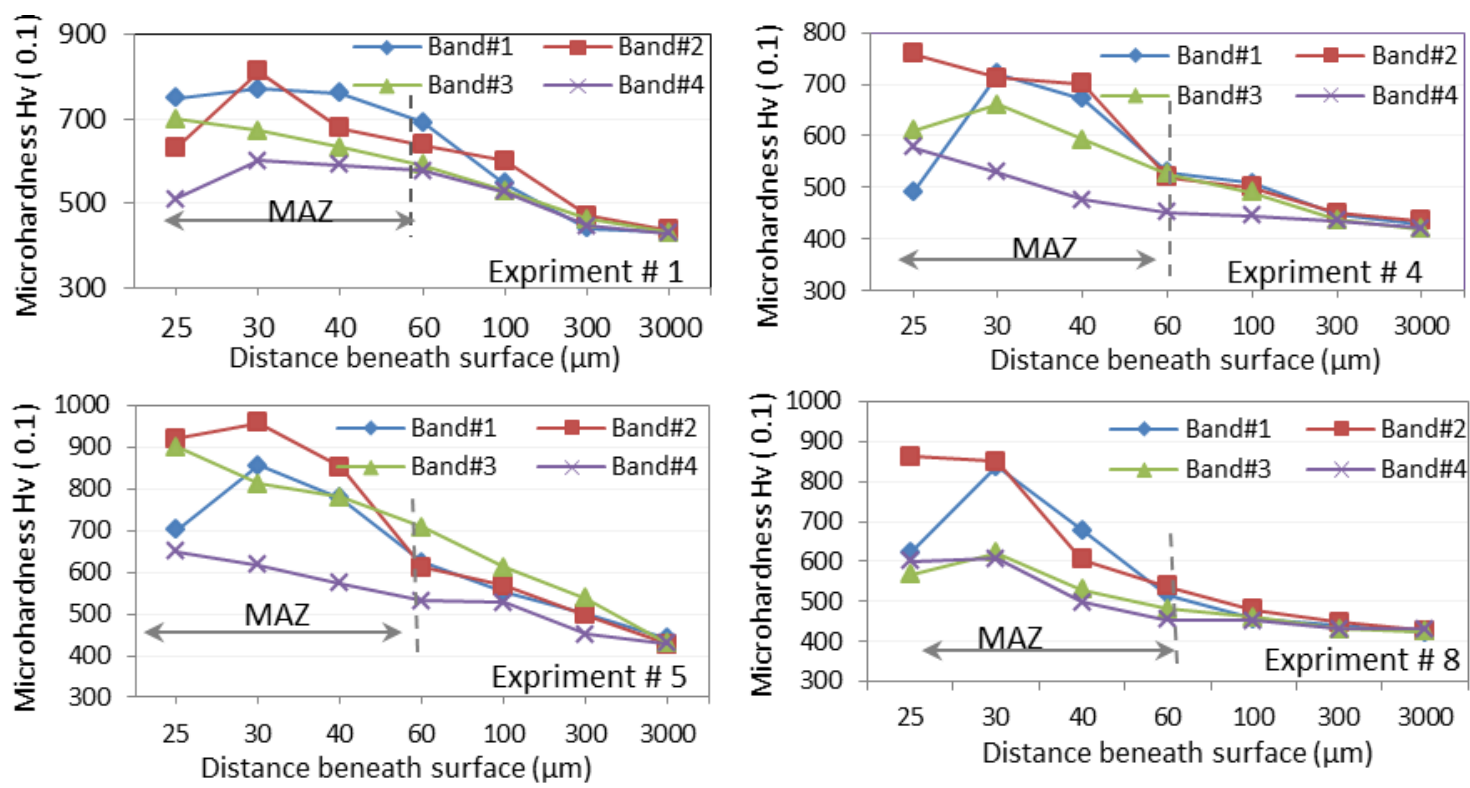

Fig. 5: Analysis of Microhardness beneath the Surface with Different Band

\section{Conclusions}

The following conclusions can be drawn from the experimental investigation of machining alteration and machining near the surface and subsurface of Inconel-718 after the machining.

- The machined surface topography revealed distinct band formation on the surface. In most of the cases, three distinct bands were observed. However, based on the measurement of surface roughness, the bands \#2 and \#3 are sometimes distinctly evident.

- The poor surface finish of $6.12 \mu \mathrm{m}$ Ra is obtained in the band \#1, an unstable cutting zone near the tool tip region. It is followed by the region of the better surface finish $(0.19 \mu \mathrm{m} \mathrm{Ra})$ in the stable cutting zone i.e. at band \#3. Again, a slightly poor surface finish at band \#4 as compared to the surface roughness Ra at band \#3 in stable cutting zone is obtained at the narrow and unstable cutting region near the periphery of the cutter.

- The microhardness variation is observed from up to $60 \mu \mathrm{m}$ beneath the ball end milled surface.

- A higher microhardness was obtained very close to the machined surface at $25 \mu \mathrm{m}$ depth due to mechanically dominated effect in the form of severe plastic deformation than thermal effect or rapid heating and quenching. The microhardness gradient becomes linear with an increase in the depth beneath the machined surface, which indicates that the plastic deformation gradually weakens with an increase of the depth beneath the surface.

\section{Acknowledgments}

Authors gratefully acknowledge the help provided by Sunil Sanap during the experimental work. Authors are grateful towards the guidance and help provided by Harshad Sonawane during testing of work specimen. Thanks are due to MHRD Govt. of India, TEQIP-I for providing the grant for CNC milling machine for the experimental work.

\section{References}

[1] R. Arunachalam, M. A. Mannan. Machinability of nickel-based high temperature alloys. J Mach Sci and Technol, 2000, 4, 127-168. http://dx.doi.org/10.1080/10940340008945703.

[2] E. O. Ezugwu, J. Bonney, Y. Yamane .An overview of the machinability of aero Engine alloy. J Mate Process Technol, 2003, 135, $233-253$. http://dx.doi.org/10.1016/S0924-0136(02)01042-7.

[3] Harshad A. Sonawane, Suhas S. Joshi Analytical modeling of chip geometry and cutting forces in helical ball end milling of super alloy Inconel 718. CIRP Manuf Technol, 2010, 3, 204. http://dx.doi.org/10.1016/j.cirpj.2010.11.003.

[4] Irfan Ucun, KubilayAslantas, FevziBedir, An experimental investigation of the effect of coating material on tool wear in micro milling of Inconel 718. Wear, 2013, 300, 8-19. http://dx.doi.org/10.1016/j.wear.2013.01.103.

[5] M.C. Shaw Metal Cutting Principles. Clarendon press Oxford Magazine, 17, 1947.

[6] Nandkumar N. Bhopale, Raju S. Pawade \& Suhas S.Joshi Experimental Investigation into the effect of Ball end milling parameters on Surface Integrity of Inconel 718. J of Mate Engg and Perform, 2014, 23.

[7] R.S. Pawade, S.S. Joshi, P.K. Brahmankar. Effect of cutting edge geometry and maching parameters on surface integrity of high-speed turned Inconal 718. J Mach Tools and Manuf, 2008, 48, 15-28. http://dx.doi.org/10.1016/j.ijmachtools.2007.08.004.

[8] Aspinwall DK. Sharman RC. High speed ball nose end milling of Inconel 718. ClRP Ann Manuf Technol, 2001, 49, 41-46.

[9] K.D. Bouzakis, P. Aichouh, K. Efstathiou. Determination of the chip geometry, cutting force and roughness in free form surfaces finishing milling with ball end tools. J Mach Tools Manuf, 2003, 43, 499-514. http://dx.doi.org/10.1016/S0890-6955(02)00265-1. 
[10] Harshad A. Sonawane, Suhas S. Joshi. Analysis of machined surface quality in a Single-pass of ball-end milling on Inconel 718. J Manuf Process, 2012, 14, 257 - 268. http://dx.doi.org/10.1016/j.jmapro.2012.03.003.

[11] Mizugaki Y, Kikkawa K, Terai H, Hao M. Theoretical estimation of machined surface profile based on cutting edge movement and tool orientation in ball nosed end milling. CIRP Ann, 2003, 52, 49-52. http://dx.doi.org/10.1016/S0007-8506(07)60528-9.

[12] A. Shokrani, V. Dhokia, S.T Newman, R. Imani-Asrai. An initial study of the effect of using liquid nitrogen coolant on the surface roughness of Inconel 718 in CNC milling. CIRP, 2012, 3, 121 -125. http://dx.doi.org/10.1016/i.procir.2012.07.022.

[13] M.S. Kasim, C.H.CheHaron, J.A.Ghani, M.A.Sulaiman, M.Z.A.Yazid. Wear mechanism and notch wear location prediction model in ball nose end milling of Inconel 718. Wear, Article in press, 2013. http://dx.doi.org/10.1016/j.wear.2012.12.040.

[14] G.K. Dosbaeva, S.C. Veldhuis, A. Elfizy, G. Fox-Rabinovich, and T. Wagg. Microscopic observations on the origin of defects during machining of direct aged Inconel 718. Journal of Materials Engg and Performance, 2010, 19, 1193-1198. http://dx.doi.org/10.1007/s11665$\underline{009-9587-3 .}$.

[15] Bulent Kaya, Cuneyt Oysu, Huseyin M. Ertunc. Force-torque based on-line tool wear estimation system for CNC milling of Inconel 718 using neural networks. Adv engg soft, 2011, 42, 76-84.

[16] Kennametal Master Tooling Catalog. Sustainable solution for Aerospace Manufacturing and Advance Material for a new generation, 2012.

[17] D.C. Montgomery. Design and Analysis of Experiments. John Wiley and Sons, New York, 4th Edition, 1997.

[18] Brian Griffiths. Manufacturing Surface Technology. Elsev Sci and Technol 1st Edition, 2001.

[19] S. Zhang, T. C. Ding and J. F. Li. Microstructural alteration and microhardness at near surface of AISI H13 steel by hard milling. J Mach Sci and Technol, 2012, 16, 473-486. http://dx.doi.org/10.1080/10910344.2012.699387.

[20] Bhopale N.N. and R. S. Pawade. Investigation of SurfaceIntegrity in High-speed Ball End Milling of Cantilever Shaped Thin Plate of Inconel 718. Journal of Achievements in Materials and Manufacturing Engineering, 2012, 55, 616-622. 